\title{
Structure of the Holliday junction intermediate in Cre-loxP site-specific recombination
}

\author{
Deshmukh N.Gopaul, Feng Guo and \\ Gregory D.Van Duyne ${ }^{1}$
}

\author{
Department of Biochemistry and Biophysics and Johnson Research \\ Foundation, University of Pennsylvania School of Medicine, \\ Philadelphia, PA 19104, USA \\ ${ }^{1}$ Corresponding author
}

We have determined the $\mathrm{X}$-ray crystal structures of two DNA Holliday junctions (HJs) bound by Cre recombinase. The $\mathrm{HJ}$ is a four-way branched structure that occurs as an intermediate in genetic recombination pathways, including site-specific recombination by the $\lambda$-integrase family. Cre recombinase is an integrase family member that recombines 34 bp loxP sites in the absence of accessory proteins or auxiliary DNA sequences. The $2.7 \AA$ structure of Cre recombinase bound to an immobile $\mathrm{HJ}$ and the $2.5 \AA$ structure of Cre recombinase bound to a symmetric, nicked $\mathrm{HJ}$ reveal a nearly planar, twofold-symmetric DNA intermediate that shares features with both the stacked-X and the square conformations of the $\mathrm{HJ}$ that exist in the unbound state. The structures support a protein-mediated crossover isomerization of the junction that acts as the switch responsible for activation and deactivation of recombinase active sites. In this model, a subtle isomerization of the Cre recombinase-HJ quaternary structure dictates which strands are cleaved during resolution of the junction via a mechanism that involves neither branch migration nor helical restacking. Keywords: Cre recombinase/Holliday junction/ recombination

\section{Introduction}

Genetic recombination results in the exchange of information between DNA segments and plays a central role in the maintenance and propagation of genomes. In eukaryotes, for example, meiotic recombination generates diversity by exchanging regions of homologous chromosomes and serves as a mechanical organizer in preparation for chromosome segregation (Kleckner, 1996). In bacteria, homologous recombination is a key component of conjugation and DNA-repair pathways (Kowalczykowski et al., 1994). A site-specific recombination process is responsible for integration and excision of bacteriophage genomes into and out of their bacterial host chromosomes (Campbell, 1962; Weisberg and Landy, 1983). Although the enzymatic details of the early steps involved in these recombination reactions differ, and are in some cases not yet well understood, each case involves a common fourway branched DNA intermediate known as the Holliday junction (Holliday, 1964).
A Holliday junction (HJ) is formed when a single strand from each of two duplex DNA segments involved in recombination is exchanged in a region of sequence homology to yield a branched structure that has four duplex arms. An important property of the $\mathrm{HJ}$ is its ability to undergo branch migration through regions of sequence homology; this isomerization process involves the simultaneous melting and annealing of base pairs and results in movement of the branch point along the arms of the junction (Meselson, 1972). The ultimate fate of HJs in vivo is cleavage of two of the DNA single strands to yield unbranched products. Depending on the extent of branch migration and which pairs of strands are cleaved, the nature of the duplex products can be quite different. A number of HJ-resolving enzymes that differ in sequence and strand specificity of cleavage have been identified from bacteria (Dunderdale et al., 1991; Iwasaki et al., 1991; Sharples et al., 1994), bacteriophages (Mizuuchi et al., 1982; de Massey et al., 1984), yeast (Symington and Kolodner, 1985; West and Korner, 1985; Kleff et al., 1992) and higher eukaryotes (Elborough and West, 1990; Sekiguchi et al., 1996). It has recently become clear that the cleavage specificities of these enzymes, and therefore the types of recombinant products generated, are intimately related to the structures adopted by the branched DNA substrates (Parsons et al., 1990; Bennett and West, 1995b; Duckett et al., 1995; Arciszewska et al., 1997; Azaro and Landy, 1997).

We have been studying the structures of intermediates in the site-specific recombination pathway of bacteriophage P1 Cre recombinase (Cre) (Abremski et al., 1983). Cre is a member of the bacteriophage $\lambda$-integrase family of recombinases, which also includes the Flp recombinase from yeast (Sadowski, 1995), the XerC/XerD recombinases from Escherichia coli (Blakely et al., 1993) and $>100$ other members identified from sequence analyses (Argos et al., 1986; Nunes-Düby et al., 1998). Members of this large family of enzymes perform a variety of genetic manipulations. $\lambda$-integrase catalyzes the integration and excision of the phage $\lambda$ genome to and from the host chromosome (Weisberg and Landy, 1983; Landy, 1989); Cre and the XerC/XerD recombinases maintain circular DNA replicons in a monomeric state (Sternberg et al., 1981; Sherratt et al., 1995) and Flp recombinase mediates the amplification of $2 \mu$ plasmid copy number by inverting a DNA segment during replication (Futcher, 1986).

The $\lambda$-integrase (Int) family shares a common mechanism for carrying out site-specific recombination that involves the formation of a HJ intermediate (Craig, 1988; Stark et al., 1989). The reaction is shown schematically in Figure 1A. A nick is produced in each of the two DNA substrates when conserved tyrosine side chains from two recombinase subunits cleave at specific sites, forming 3'- 
A
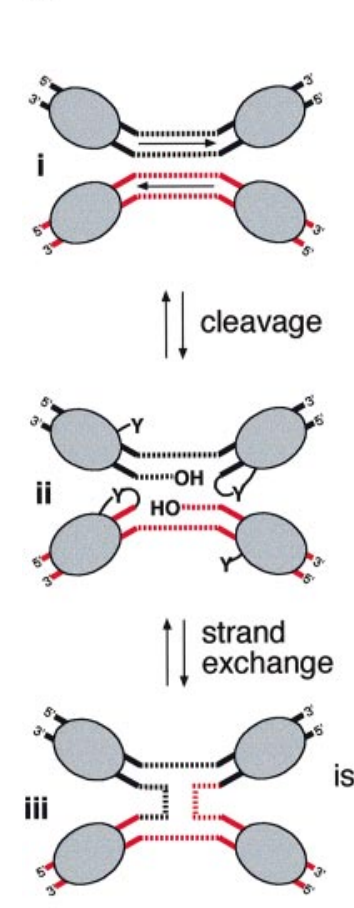
is somerization $\longleftrightarrow$

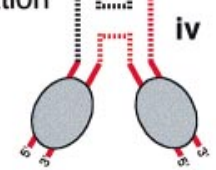

B

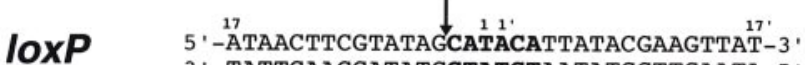
3 ' -TATTGAAGCATATCGTATGTAATATGCTTCAATA-5'

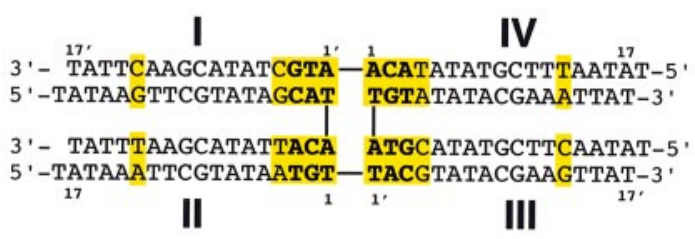

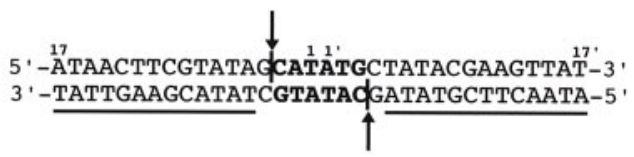

cleavage

IoxS6

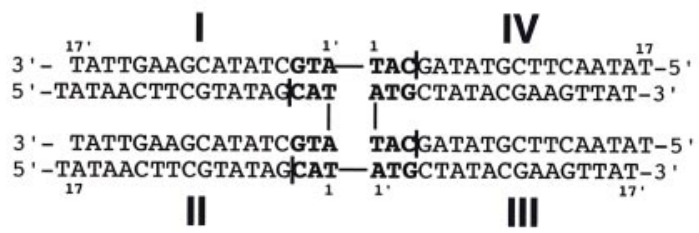

Fig. 1. (A) Schematic drawing of the Cre-loxP site-specific recombination pathway, based on the strand-swapping model (Nunes-Düby et al., 1995) and on Cre-loxP structural models (Guo et al., 1997; this work). Conserved tyrosine residues from two of the four recombinases in a synaptic tetramer cleave the DNA backbones of the recombining segments to form transient 3'-phosphotyrosine linkages. The released 5 '-hydroxyl ends of the cleaved DNA undergo intermolecular nucleophilic attack of the partner phosphotyrosine linkages to complete the exchange of one pair of strands and form a Holliday intermediate. A second round of cleavages and strand exchanges using the remaining two recombinase subunits and the complementary DNA strands gives recombinant products. (B) Sequences of loxP and loxS6 DNA duplexes used to design the HJs HJ1 and HJ2. HJ1 and $\mathrm{HJ} 2$ are shown in the same orientation as in Figure 3. For HJ1, the strand-bridging arms I and II contain the wild-type loxP sequence and the strand-bridging arms III and IV contain the loxP complementary sequence. Bases that are not related by twofold symmetry and prevent branch migration are highlighted in yellow. Vertical lines indicate missing phosphates in the DNA backbone. The 13 bp inverted repeat binding sites for Cre recombinase are underlined and the $6 \mathrm{bp}$ crossover region between cleavage sites are in bold. For both the Cre-HJ1 and Cre-HJ2 complexes, an additional 5'-overhanging thymidine residue was found to facilitate crystallization.

phosphotyrosine linkages with the substrates and releasing free 5'-hydroxyl DNA ends. The 5'-hydroxyl groups carry out intermolecular nucleophilic attack of the partner phosphotyrosine linkages to complete the exchange of one set of strands and produce a Holliday intermediate. The Holliday structure is then resolved to the recombinant products by cleavage and exchange of the remaining pair of strands by the second pair of recombinase subunits. Structural and mechanistic similarities between the Int family and the eukaryotic topoisomerase I family of enzymes have recently been demonstrated (Cheng et al., 1998).

Cre recombinase is a $38.5 \mathrm{kDa}$ protein that catalyzes site-specific recombination between 34 bp loxP sites. The loxP sequence (Figure 1B) is composed of two $13 \mathrm{bp}$ recombinase-binding elements arranged as inverted repeats which flank an $8 \mathrm{bp}$ central region where cleavage and ligation reactions occur (Hoess et al., 1982). This core recombination site organization is common to the Int family members that have been well-studied, although the length of the binding sites and central segments vary and some enzymes require auxiliary DNA sequences outside of this core region. Recent investigations into the mechanism of Int family site-specific recombination have focused on the nature of the recombinase-bound Holliday intermediate, and in particular on the ability of the DNA junction conformation to influence the direction of resolution to products (Arciszewska et al., 1997; Azaro and Landy, 1997; Lee et al., 1998).

Previously we described the structure of a covalent intermediate in the Cre-loxP site-specific recombination pathway (Figure 1A, ii and v), where two of the four catalytic tyrosine residues had cleaved the DNA substrates and formed 3'-phosphotyrosine linkages (Guo et al., 1997). This synaptic complex was trapped at the strand exchange step of the reaction. Here we report two X-ray crystal structures of Cre recombinase-HJ (Cre-HJ) complexes, representing the pivotal intermediate in the Cre-loxP site-specific recombination pathway (Figure 1A, iii and iv). The first, determined at $2.7 \AA$ resolution, contains an immobile junction with the branch point fixed at the center of the crossover region. The second, determined at $2.5 \AA$, contains a partially mobile junction formed from nicked DNA substrates. Both structures indicate that the Cre-bound $\mathrm{HJ}$ adopts a pseudoplanar, dyad-symmetric conformation distinct from either the stacked-X or the extended square models that have been deduced from studies of isolated junctions (Duckett et al., 1988). The Cre-HJ structures also suggest a model for isomerization of the recombinase-HJ complex that involves a more subtle rearrangement of protein and DNA quaternary structure than previously proposed. 


\section{Results}

\section{Construction of Cre-HJ complexes}

To construct a HJ substrate for Cre recombinase, four 35-mer oligodeoxynucleotides were annealed to give the junction HJ1 shown in Figure 1B. The sequences of the junction arms are modifications of the wild-type loxP site. Because a $\mathrm{HJ}$ generated from two 34 bp loxP sites contains dyad symmetry with respect to the sequences of the arms and would spontaneously branchmigrate into duplex molecules in solution, it was necessary to introduce asymmetry into the arm sequences of HJ1 (Kallenbach et al., 1983). The HJ1 arms were designed by using the wild-type loxP-crossover sequence for arms I and II, but swapping top and bottom strands of the loxP-crossover sequence in arms III and IV. In addition, $1 \mathrm{bp}$ was changed in each of the inverted repeat regions of the arms. This site was chosen by inspection of the Cre recombinase-DNA (Cre-DNA) interface observed in the structure of a synaptic CreDNA covalent intermediate (Guo et al., 1997) and was predicted to tolerate change to any of the four DNA base pairs. Together, the asymmetric crossover region and the unique base pair located in each of the inverted repeat arms of the junction effectively destabilizes formation of alternative secondary structures from the single-stranded components and yields an immobile junction.

In order to prevent cleavage of $\mathrm{HJ} 1$ by the recombinase, a Cre R173K mutant was used in the structure analysis. Arginine-173 is a strictly conserved active-site residue in the Int family (Abremski and Hoess, 1992) that participates in activation of the scissile phosphate. Mutations at this site render Cre inactive in the complete recombination reaction (Wierzbicki et al., 1987) and unable to cleave the loxA suicide DNA substrate (Guo et al., 1997; data not shown). We chose not to use inactive Cre mutants involving changes to the conserved nucleophile Tyr324 for these structural studies. In our previous covalent CreDNA complex structure and in the Cre-HJ structures described here, Tyr324 makes a hydrogen bond adjacent to the scissile phosphate and this interaction may be an important component of active-site assembly prior to cleavage.

The symmetric junction $\mathrm{HJ} 2$ was derived from a pair of symmetrized loxP sites containing nicks at the positions of the scissile phosphates. To symmetrize the loxP site, three positions in the crossover region were changed to give the sequence shown in Figure 1B. This sequence is identical to the loxA suicide substrate used to prepare covalent Cre-DNA intermediates (Guo et al., 1997), except the nick has been moved to the position of the scissile phosphate. The loxS6 HJ precursor was formed by annealing two oligodeoxynucleotides to form duplexes with 6 bp self-complementary overhangs which dimerize to give the full site. Following Cre binding and synapsis of the loxS6 sites, strand exchange between duplexes occurs in the absence of ligation, leading to formation of a HJ complex (see Discussion). Wild-type Cre recombinase was used in complex with HJ2, since the phosphodiester linkages normally attacked by Cre are missing in this substrate.
Table I. Summary of crystallographic refinements

\begin{tabular}{lcc}
\hline & Cre-HJ1 & Cre-HJ2 \\
\hline Resolution & $48-2.7 \AA$ & $27-2.5 \AA$ \\
$\mathrm{R}_{\text {symm overall } / \mathrm{R}_{\text {symm }} \text { high res shell }}$ & $0.072 / 0.33$ & $0.053 / 0.25$ \\
Completeness/redundancy & $99 \% / 4.7$ & $94 \% / 2.8$ \\
R-working/ $\mathrm{R}_{\text {free }}(\mathrm{F} \geqslant 2 \sigma)$ & $0.22 / 0.29$ & $0.20 / 0.27$ \\
Refined model: & & \\
$\quad$ Protein residues & $622 / 686$ & $614 / 686$ \\
DNA bases & $68 / 70$ & $68 / 70$ \\
$\quad$ Solvent & 239 & 361 \\
r.m.s. bonds & $0.005 \AA$ & $0.007 \AA$ \\
r.m.s. angles & $0.95^{\circ}$ & $1.3^{\circ}$ \\
\hline
\end{tabular}

\section{Structure of the Cre-HJ complex}

Structures of the Cre-HJ1 and Cre-HJ2 complexes were determined at $3 \AA$ resolution and refined to final resolutions of 2.7 and $2.5 \AA$, respectively (see Materials and methods; Table I). The two complexes have nearly identical structures, despite sequence differences near the branch point of the DNA junctions and the presence of four nicks in the phosphate backbone of HJ2. The root mean square (r.m.s.) difference in $\alpha$-carbon positions between the two different complexes is 0.63 and $0.60 \AA$ for the subunits bound to DNA arms I and II, respectively. The structure of the Cre-HJ2 complex is shown in Figure 2. The Cre$\mathrm{HJ} 1$ complex is indistinguishable from Cre-HJ2 in these drawings (not shown). One Cre recombinase protein binds to each of the four arms of the HJ, forming a 'C-clamp' around the inverted repeat binding sites (base pairs 5-17). The N-terminal domain covers one face of the duplex and the C-terminal domain covers the opposite face (Figure 2D). These Cre-DNA arm complexes form a pseudofourfold-symmetric tetramer through an extensive network of protein-protein interactions involving both the $\mathrm{N}$ - and C-terminal domains. The Cre-DNA interfaces in the arms are all similar to one another and to that seen in the covalent Cre-loxA complex (Guo et al., 1997). The protein-protein interactions in the Cre-HJ complexes are also similar to those observed in the Cre-loxA structure, including a cyclic exchange of C-terminal $\alpha$-helices (helix $\mathrm{N}$ ) between adjacent subunits (Figure 2C). The Cre-HJ1 and Cre-HJ2 complexes have a crystallographic dyad axis that relates opposite halves of the structures. The r.m.s. difference between well-ordered $\alpha$-carbon atoms in the two unique recombinase subunits is $1.7 \AA$ for the Cre$\mathrm{HJ} 1$ complex and $1.5 \AA$ for Cre-HJ2.

\section{Structure of the Cre recombinase-bound HJ}

The most conspicuous features of the Cre recombinasebound $\mathrm{HJ}$ are the relative dispositions of the junction arms and the unstacking of base pairs at the branch point (Figure 3). The complexes have exact twofold symmetry, but only approximate fourfold symmetry. For example, the angle between arms I and II is $76^{\circ}$ and the angle between arms II and III is $101^{\circ}$. This gives rise to a pronounced distortion from a fourfold-symmetric structure that would have equivalent inter-arm angles (see Materials and methods for definitions of geometric parameters). The Cre-HJ arms do not form pairs of coaxially stacked helices as shown for unbound junctions in the presence of physiological salt concentrations (Duckett et al., 1988), 
A
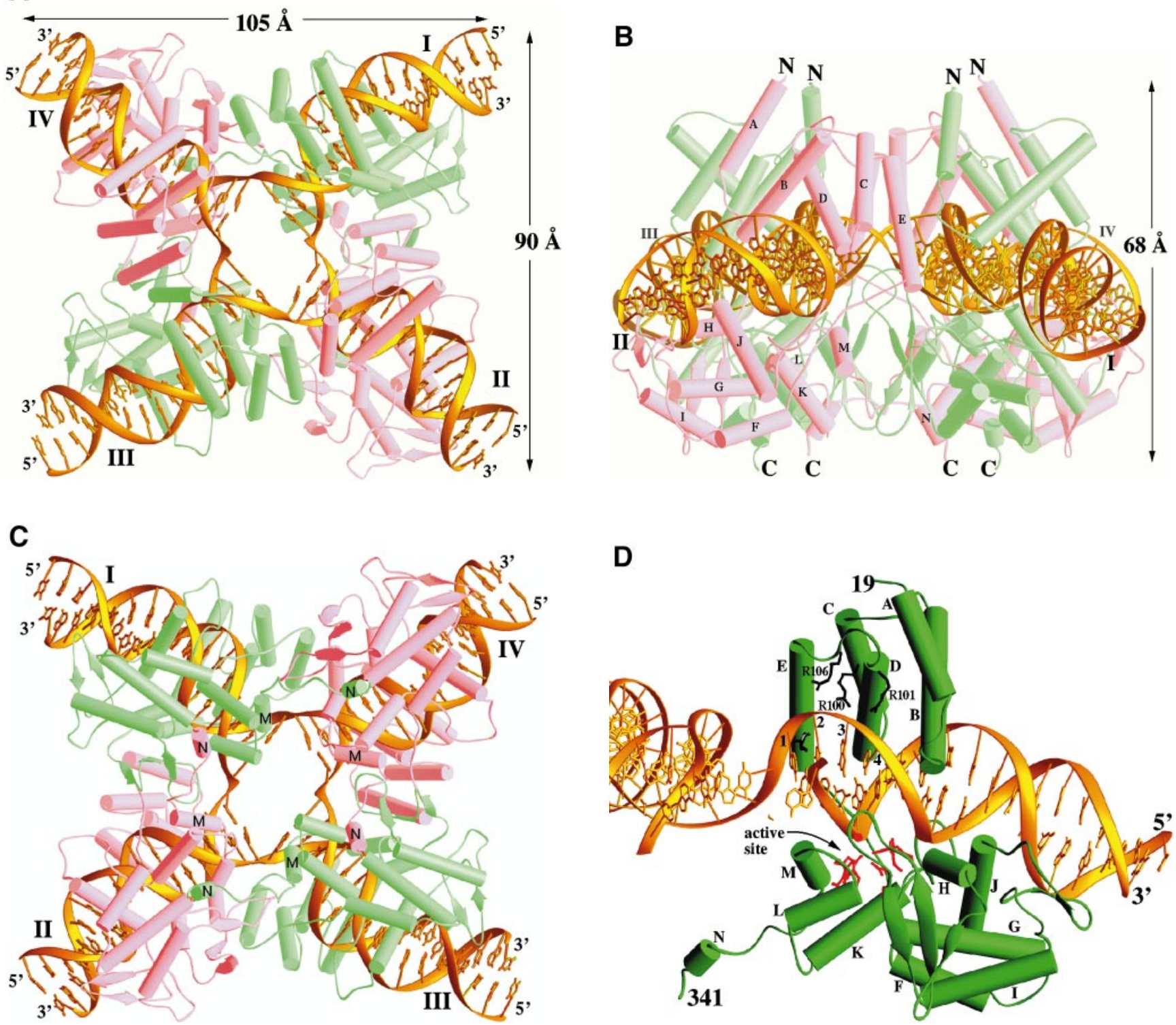

Fig. 2. Structures of the Cre-HJ1 and Cre-HJ2 complexes. Because the complexes are very similar, only the Cre-HJ2 structure is shown, with continuous DNA ribbons drawn through the missing phosphates. (A) View from the N-terminal recombinase domain side and the minor groove face of the junction. (B) Side view illustrating the near planar arrangement of the DNA arms. Helices of one recombinase protomer are labeled. (C) View from the C-terminal recombinase domain side and the major groove face of the junction. C-terminal helices $\mathrm{M}$ and $\mathrm{N}$, which are involved in a cyclic exchange of $\alpha$-helices between recombinase protomers, are labeled. (D) One recombinase protomer bound to a single junction arm. The active site residues are in red and the position of the scissile phosphate is represented by a red sphere. Arginine residues that contact phosphates near the branch point are shown in black.

but instead have sharp discontinuities in stacking at the branch point. The DNA bases located at the branch point all make Watson-Crick base pairs, but have become unstacked, with local tilt angles of $-113^{\circ}$ between base pairs bridging arms I and II and $-65^{\circ}$ for base pairs between arms II and III. The same tilt angles with respect to a global helicoidal axis through neighboring arms are -110 and $-60^{\circ}$, respectively.

Although the HJ adopts an approximately planar conformation, a small out-of-plane bend in the junction arms creates a concave surface on the face of the junction that is contacted by the $\mathrm{C}$-terminal catalytic domains of the recombinase tetramer (Figure 2B). This is also the face of the DNA junction where the major grooves of the four arms converge (Figure 3). The cleavage and ligation steps in the Cre recombinase site-specific recombination reaction therefore occur on the major groove face of the four-way junction substrate. The opposite minor groove face of the junction is bound by the N-terminal recombinase domains. The extent of bending with respect to the best plane through the DNA junction differs for the two unique arms in the Cre-HJ complexes. Arm I makes an angle of $13^{\circ}$ with the plane and an angle of $153^{\circ}$ with its dyadsymmetric arm III, whereas arm II is $8^{\circ}$ from the plane and makes an angle of $164^{\circ}$ with arm IV. The distortion from a fourfold-symmetric junction is therefore not limited to rotational differences about an axis perpendicular to the plane of the junction, but also includes small out-ofplane bends that differ for neighboring arms. Within the arms of the junction, the helicoidal DNA parameters 
A
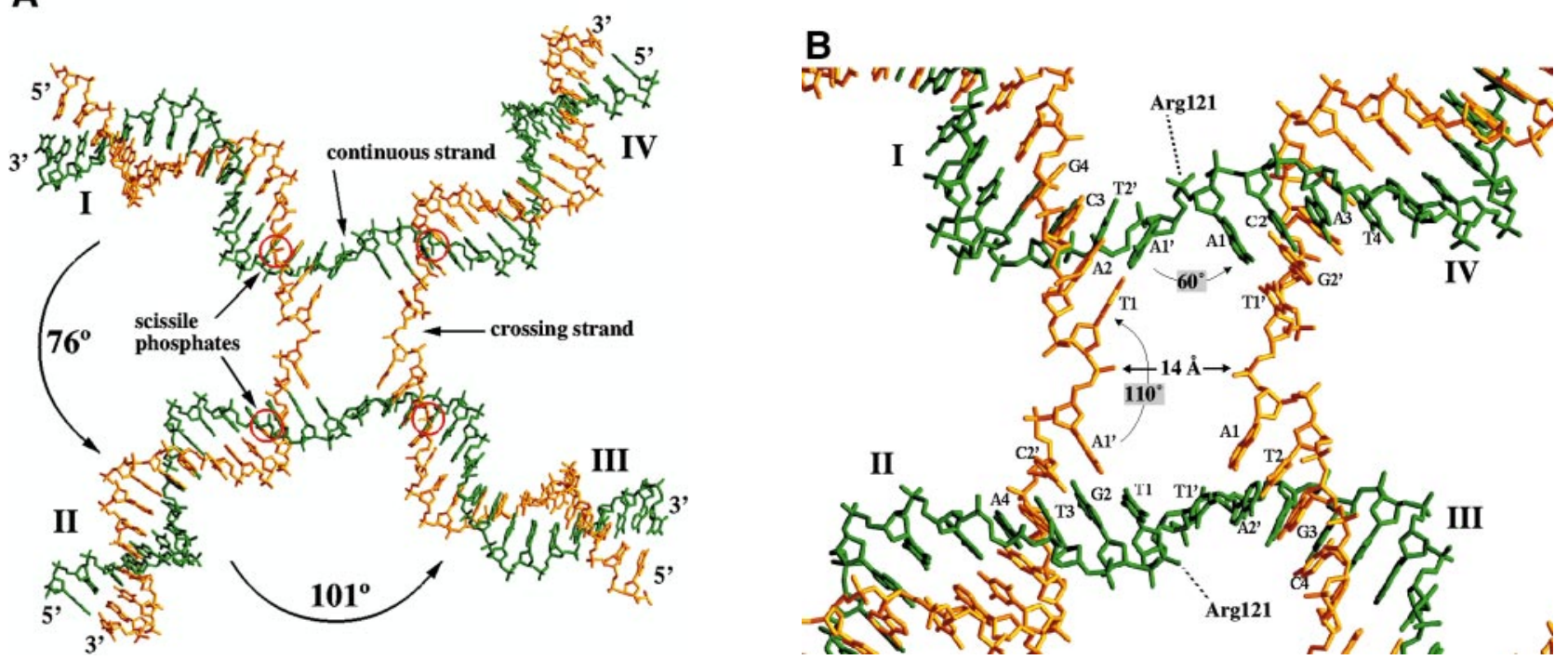

Fig. 3. Structure of the Cre recombinase-bound HJ. The HJ1 structure is shown, with the arm sequences as in Figure 1 (see Materials and methods). (A) Overall geometry of the four-way junction. (B) Close-up of the center of the junction, where angles refer to helicoidal tilt angles as described in the text.

are consistent with those found in moderately bent DNA duplexes in high resolution crystal structures of proteinDNA complexes. A bend of $\sim 20^{\circ}$ is smoothly distributed throughout the $13 \mathrm{bp}$ binding sites of each arm and is responsible for the concave DNA surface on the major groove face of the junction. DNA arms I and II have similar structures, with r.m.s. differences of $1.6 \AA$ within both the Cre-HJ1 and Cre-HJ2 complexes, and a maximum of $0.8 \AA$ for pairwise comparison of the same arms between the two complexes.

The source of the sharp bends between junction arms I and II is different than for arms II and III. For arms II and III, the torsion angle about the $\mathrm{C}^{\prime}-\mathrm{O}^{\prime}$ bond $(\varepsilon)$ for the $1-1^{\prime}$ phosphodiester linkage is $-87^{\circ}$ instead of the trans conformation typical of a B-DNA duplex. For arms I and II, the $\varepsilon$ torsion angle is trans, but the $\mathrm{P}-\mathrm{O}^{\prime}$ torsion angle $(\zeta)$ and the $\mathrm{P}-\mathrm{O} 5^{\prime}$ torsion angle $(\alpha)$ are +44 and $+58^{\circ}$, respectively. Both are close to a gauche $^{+}$ conformation, whereas the same torsion angles in standard B-DNA are approximately -90 and $-60^{\circ}$, respectively. In addition to creating a sharp bend at the junction branch point, the $g^{+} / g^{+}$conformation for torsion angles $\zeta$ and $\alpha$ causes the phosphate group to swivel $180^{\circ}$ towards the center of the junction. This phosphate inversion is evident in Figure 3, which shows the two pairs of dyad-related non-bridging phosphate oxygen atoms facing one another across the center of the junction with a separation of about $14 \AA$ A.

The unstacking of bases at the junction branch point causes a dramatic increase in solvent accessibility to the planar purine and pyrimidine surfaces. Both the Cre $\mathrm{N}$-terminal domain tetramer on the minor groove face of the junction and the C-terminal domain tetramer on the major groove face form channels that are large enough to allow solvent access to the junction branch point (Figure 4). An average of $\sim 170 \AA^{2}$ of base pair surface is accessible to solvent for the four AT base pairs at the branch point in the Cre-HJ complexes. For comparison, the same base

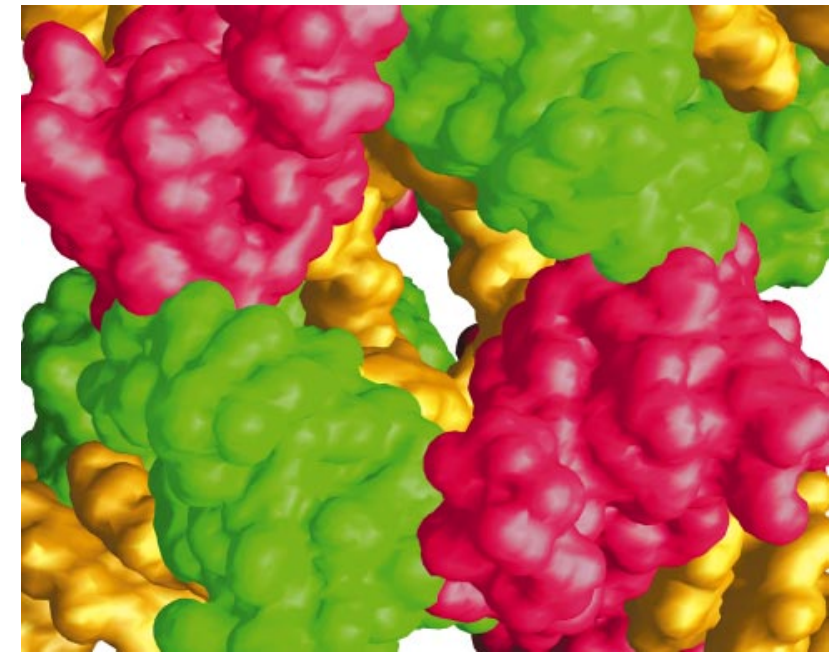

Fig. 4. Solvent accessible surface at the center of the Cre-HJ complex, as viewed from the $\mathrm{N}$-terminal, minor groove side of the junction. The surfaces of adenine and thymine bases at the branch point as well as the inverted phosphates are clearly visible through a solvent-accessible tunnel formed by the $\mathrm{N}$-terminal domains. The orientation of the complex is the same as in Figure 2A.

pair type near the center of the loxP-inverted repeat region has only $\sim 80 \AA^{2}$ of base-pair surface accessible to solvent (ignoring the bound recombinase). Chemical probing of solvent accessibility of bases at or near the branch point of four-way DNA junctions has proved to be a revealing tool in the investigation of both free junctions and proteinjunction complexes (Duckett et al., 1988; Bennett and West, 1995b; Arciszewska et al., 1997). As discussed later, the arm geometry and solvent accessibility of bases near the branch point of the $\mathrm{Cre}-\mathrm{HJ}$ complexes allow us to draw close parallels with models resulting from some studies and sharp contrasts with others.

Given the requirement for large movements of DNA 
strands within the $\operatorname{lox} P$ crossover region during the strand exchange step of the site-specific recombination pathway, it is perhaps not surprising that we observe few proteinDNA contacts in this region of the Cre-HJ structures or in the covalent Cre-loxA complex previously reported. Within the $4 \mathrm{bp}$ adjacent to the branch point in each arm of the junction, there are no direct contacts of the protein to bases in the major or minor grooves. Each arm of the junction is, however, contacted by recombinase side chains on the phosphate backbone in the crossover region. Three arginine side chains from the $\mathrm{N}$-terminal recombinase helix D (Arg100, Arg101 and Arg106) interact with the phosphates between bases 1-4 in each arm (Figure 2D). The contacts are slightly closer for the recombinase subunits bound to arms I and III than for the subunits bound to arms II and IV. In addition, the phosphate backbone of DNA residues $1^{\prime}, 2^{\prime}$ and $3^{\prime}$ is closely approached by a poorly ordered loop that includes residues Thr200-Thr206. The electron density for these residues is of insufficient quality to reliably identify DNA contacts. This loop was better-ordered in the covalent Cre-loxA complex and we were able to identify Lys201 in the minor groove of the DNA, interacting with the base pair adjacent to the scissile phosphate (Guo et al., 1997). Lys201 is conserved in the Int family, except for a small number of proteins with arginine in this position (Nunes-Düby et al., 1998).

The central phosphate between arms II and III forms the most conspicuous backbone contact within the crossover region, hydrogen bonding with Arg121 in the Cre-HJ1 complex, and interacting from a slightly larger distance $(\sim 4 \AA)$ in the $\mathrm{Cre}-\mathrm{HJ} 2$ complex (Figure 3B). An equivalent interaction is not made with the central phosphate between arms I and II, which has turned towards the center of the junction away from Arg 121. As a consequence of the lack of hydrophobic and major-minor groove interactions within the crossover region, the mobility of DNA in the center of the junction is increased as reflected in larger average thermal parameters $(<\mathrm{B}\rangle=49 \AA^{2}$ in the crossover region versus $35 \AA^{2}$ in the 13 bp inverted repeat region of the Cre-HJ1 complex) following crystallographic refinement.

\section{The active sites in the Cre-HJ intermediate}

The Cre recombinase active site contains four amino acids that are strictly conserved in the Int family (Abremski and Hoess, 1992). Three of these residues, Arg173, His289 and Arg292, make up the 'RHR triad' and form hydrogen bonds to the scissile phosphate during strand cleavage (Guo et al., 1997). The fourth conserved residue is the nucleophile Tyr324. The side chain of this residue is poised to attack the scissile phosphate, hydrogen bonding to the neighboring phosphate that lies at the boundary of the $13 \mathrm{bp}$ inverted repeat of the $\operatorname{lox} P$ site. $\operatorname{Trp} 315$, an amino acid that is more often represented by histidine in the Int family, also contributes to formation of the active site and forms a hydrogen bond to the scissile phosphate. A similar active site geometry was observed in the recently described structures of two eukaryotic topoisomerases (Cheng et al., 1998; Stewart et al., 1998).

The Cre-HJ1 and Cre-HJ2 complexes are only twofoldsymmetric overall, therefore the active sites formed by each of the two independent recombinases (i.e. the green and red subunits in Figure 2) might be expected to show some stereochemical differences. Indeed, during cleavage of the HJ substrate, only one pair of dyad-related Tyr324 side chains will cleave the DNA to initiate strand exchange. The geometric arrangement of residues in both active sites of the Cre-HJ1 and both active sites of the Cre-HJ2 complex are nevertheless quite similar, with r.m.s. deviations of $0.5 \AA$ for $\alpha$ carbon atoms. There are modest differences in side chain torsion angles among the active sites in the two complexes, but it is not yet clear whether these have functional relevance, given the missing scissile phosphates in the Cre-HJ2 structure and the altered DNA sequence used in Cre-HJ1.

The largest difference between the Cre-HJ1 active sites and the active site observed in a covalent Cre-loxA complex where cleavage had not occurred (Guo et al., 1997) involves the scissile phosphates in arms II and IV. The trajectories of the DNA strands are slightly different in the HJ complex and result in an $\sim 1 \AA$ shift in the position of the scissile phosphates. The consequences of this shift are an increase in the Tyr324-OH to phosphate distance from $5 \AA$ observed in arms I and III to $6 \AA$ in arms II and IV, and the removal of this phosphate group from ideal hydrogen bonding position. It will be important to verify this observation with further studies using alternative DNA sequences and other inactive Cre mutants in order to establish this phosphate relocation as a component of the stereochemical switch used to deactivate cleavage of a given pair of strands in the Holliday intermediate.

Although the active site geometries are similar for the two unique arms in the Cre-HJ complexes, the conformations of the linker peptides between the two C-terminal $\alpha$-helices $M$ (residues 319-326) and N (residues 333-340) adjacent to the active sites are quite different. Helix $\mathrm{N}$ is buried in a hydrophobic pocket of a neighboring recombinase subunit, forming one of the four cyclic exchanges of helices that link the arms of the recombinase-junction tetramer on the $\mathrm{C}$-terminal side of the junction (Figure 2C). Catalytic Tyr324 is located at the end of helix $M$ and this entire helix shifts by $\sim 3 \AA$ during cleavage of the DNA substrate relative to the same helix in the non-cleaving active site (Guo et al., 1997). The conformation and flexibility of the peptide segment tethered to helix $\mathrm{M}$ could therefore play a crucial role in regulating cleavage activity. This linker adopts a compact and well-ordered conformation in the connection between arms I and II of the junction and a more extended and poorly ordered conformation between arms II and III.

\section{Discussion}

\section{Intermediates in Cre-loxP recombination have similar architectures}

In this report, we have described the structures of two Cre-HJ complexes that together provide a model for this intermediate in the Cre-loxP site-specific recombination pathway. Previously, we described the structure of a covalent Cre-DNA intermediate (Figure 1A, ii and v) in which two of the four recombinases in a synaptic tetramer of symmetrized loxP sites had cleaved a suicide DNA substrate to form 3' phosphotyrosine linkages (Guo et al., 1997). Surprisingly, the $5^{\prime}$ ends of the cleaved DNA 
strands were melted away from their complementary strands and directed towards the center of that synaptic complex, which appeared to be caught in the act of exchanging strands. Because a $5^{\prime}$ cytidine had been removed in forming the phosphotyrosine bond between Cre and DNA, the 5' strands were one base too short to reach the active sites of the partner substrates. In the CreHJ2 structure reported here, this idea has been taken one step further. The loxS6 sites used to form HJ2 (Figure 1B) contain nicks where the phosphotyrosine linkage would be formed. In addition to melting and directing the 5 '-ends of the crossing strands towards the middle of the synapse, Cre has mediated formation of a $\mathrm{HJ}$ without covalent ligation to the partner substrate. Such a structure was in fact suggested by Burgin and Nash (1995) as a possible product of Int recombination using $5^{\prime}$-bridging phosphorothioate-substituted suicide substrates.

Since the sequences of the two loxS6 sites that form $\mathrm{HJ} 2$ are identical, this protein-HJ complex could, in principle, branch-migrate by 1-2 bp. The branch point of the observed crystal structure, however, is located at the center of the sites. In contrast, the immobile HJ1 DNA substrate was pre-formed before binding by Cre and was designed to have its branch point located at the center of the crossover region. The fact that the Cre-HJ1 and CreHJ2 structures are similar to one another and to the structure of the covalent intermediate (ii) in Figure 1A indicates that on the level of quaternary structure, the recombinase tetramer pre-organizes the DNA arms for HJ formation and stabilizes a single branch-point isomer of the DNA intermediate. We cannot rule out the possibility that other branch-point isomers exist for the Cre-HJ complex during site-specific recombination and that crystallization has selected a single low energy structure for Cre-HJ2. However, the architectures of Cre-HJ1 and $\mathrm{Cre}-\mathrm{HJ} 2$ argue strongly against stable recombinase tetramers with alternative branch-point positions. In order to branch-migrate a loxP-derived $\mathrm{HJ}$ substrate with Cre recombinase-bound, the extensive protein-protein contacts ( 5000 $\AA^{2}$ of buried protein surface) that stabilize the tetramer would have to be disrupted. Alternatively, if the DNA arms were allowed to rotate within the bound recombinase domains in order to accomplish branch migration, the specific Cre-DNA interface would be disrupted. Since these possibilities seem unlikely, we would argue that energy barriers to branch migration are too high in the Cre-loxP system. However, restricted branch migration may occur more readily in the Int and Flp systems, where the spacings between cleavage sites are 1-2 bp longer and the nature of the intersubunit contacts may be somewhat different.

Recent studies in the $\lambda$-integrase, XerC/XerD and Flp systems support a model of limited branch migration in the site-specific recombination pathway. Experiments performed using immobilized HJ substrates (Dixon and Sadowski, 1993; Arciszewska et al., 1995; Lee et al., 1995; Nunes-Düby et al., 1995) led to a model that does not require branch migration of the Holliday intermediate between cleavage sites for resolution of the junction. Experiments using nicked DNA substrates (Lee and Jayaram, 1995; Zhu et al., 1995), suicide phosphorothioate-containing substrates (Burgin and Nash, 1995), and suicide half-site substrates (Nunes-Düby et al., 1997) provided evidence that homology between recombining sites is first sensed following initial strand cleavage but preceding the ligation step that completes the first round of strand exchange. We believe that the Cre-HJ2 structure represents what Burgin and Nash (1995) refer to as the 'communication' step that precedes strand ligation. The Cre-HJ2 complex also represents an intermediate in the 'strand-swapping model' of site-specific recombination proposed by Landy and coworkers (Nunes-Düby et al., 1995) where the initial swapping step has just occurred (but without ligation) and homology between three out of the six base pairs between cleavage sites has been successfully tested.

\section{The Cre recombinase-bound versus free $\mathrm{HJ}$}

The properties and behavior of free HJs have been extensively studied over the past fifteen years using a number of experimental techniques, including electrophoresis, NMR, fluorescence resonance energy transfer and electric dichroism (reviewed in Lilley and Clegg, 1993; Seeman and Kallenbach, 1994). As a result of these studies, a consensus view of the solution structure of four-way junctions has emerged (Figure 5). In the presence of physiological concentrations of magnesium ions $(\geqslant 100 \mu \mathrm{M})$, the arms of the junction are organized into two quasi-continuous duplexes that are fully stacked at the junction branch point. The helical stacks form a right-handed cross with an acute angle of roughly $60^{\circ}$, leading to an overall dyad-symmetric shape termed the 'stacked-X' (Churchill et al., 1988; Duckett et al., 1988). The pairs of arms in this four-way junction are distinctly non-coplanar (Figure 5B). Within each helical stack, a continuous strand forms an uninterrupted helix through the branch-point region. The opposite crossing strand has a sharp discontinuity in its helicoidal path at the junction branch point, where it crosses from one stack to the other. The two strands in a given arm therefore have distinctly different roles at the branch point. There are several ways of constructing such a junction; the continuous strands can be in parallel or antiparallel orientation with respect to alignment of the $5^{\prime}-3^{\prime}$ strand direction and the roles of crossing and continuous strands can be switched for each orientation, leading to strand equivalence during recombination (Sigal and Alberts, 1972). Experimental and theoretical studies indicate that the antiparallel alignment is favored (von Kitzing et al., 1990; Lu et al., 1991), but a given antiparallel junction can exist as one of two crossover isomers depending on which strand crosses between helical stacks. These crossover isomers differ by relatively small free energies that depend on the sequences present at and near the branch point and both isomers can be populated for some junction sequences (Miick et al., 1997).

In the presence of EDTA and low salt, the free HJ adopts a fourfold-symmetric square conformation, where the arms of the junction point towards the vertices of a square (Figure 5A; Duckett et al., 1988; Clegg et al., 1994). In this case, there is an unstacking of bases at the branch point which is detectable by chemical probing with osmium tetroxide. In contrast, bases at the branch point of the stacked-X junction do not show this sensitivity to oxidation. In addition to direct experimental analyses of synthetic four-way junctions, analyses of packing in crystal structures of DNA duplexes (Goodsell et al., 1995; Wood 
A

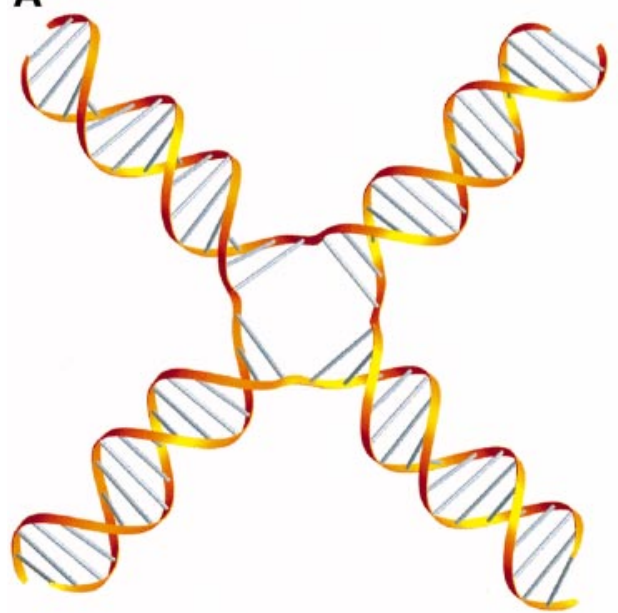

B

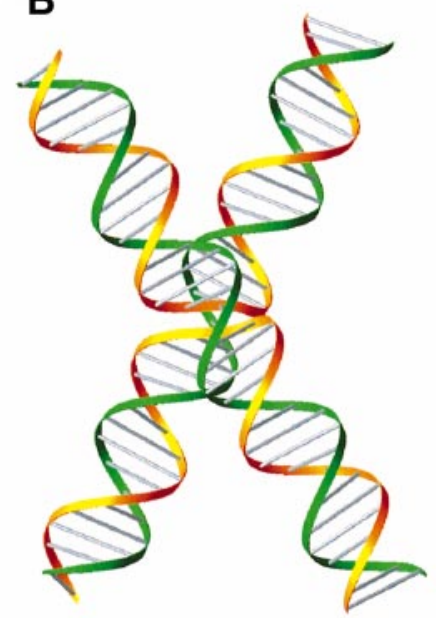

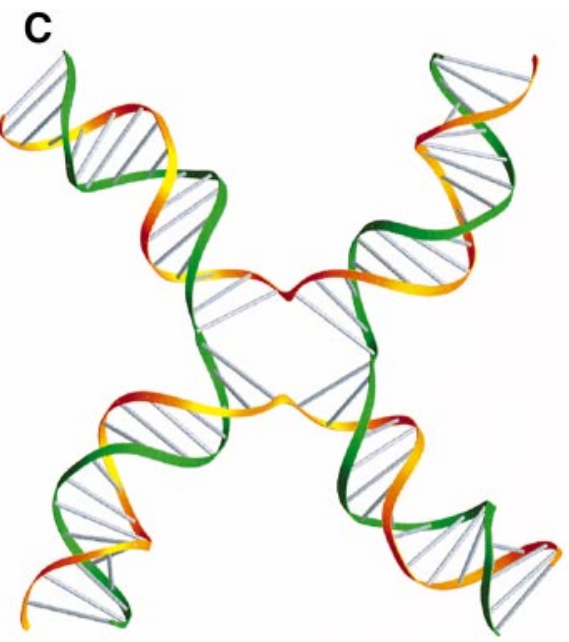

Fig. 5. Comparison of the (A) extended square, (B) stacked-X, and (C) Cre-HJ structures. Structures (A) and (B) were obtained by model-building with approximate geometries taken from theoretical models (von Kitzing et al., 1990). Continuous strands are green and crossing strands are gold for (B) and (C). The junction in (C) has been rotated $90^{\circ}$ with respect to Figure 3.

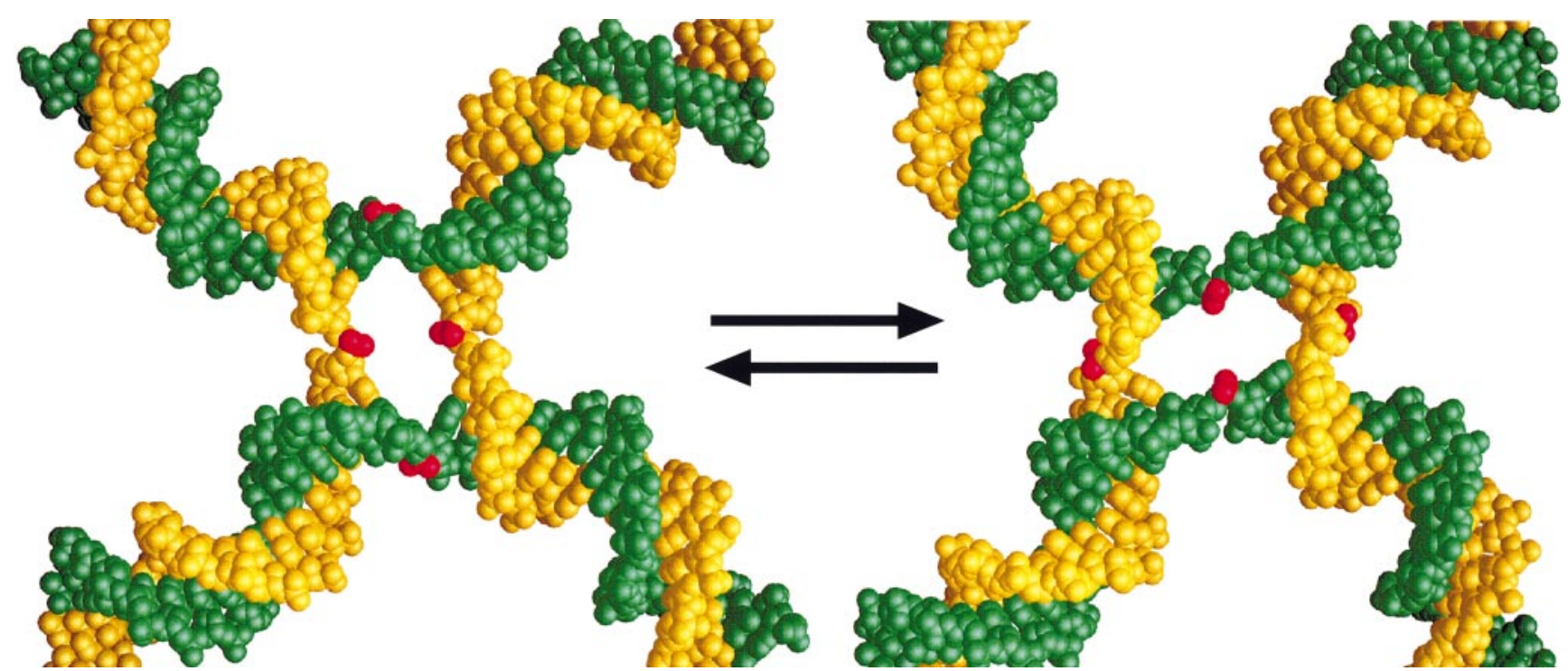

Fig. 6. Structural model for crossover isomerization of the Cre-HJ intermediate, based on the Cre-HJ1 and Cre-HJ2 complexes described here. Isomerization results in an exchange in the roles of the two pairs of 'continuous' and 'crossing' strands. This model provides a structural basis for the central isomerization step of the 'strand-swapping model' proposed by Nunes-Düby et al. (1995).

et al., 1997) and theoretical studies (von Kitzing et al., 1990) have contributed to the current models for the unbound $\mathrm{HJ}$.

The Cre-HJ1 and Cre-HJ2 complexes have features in common with both the stacked-X and the square HJ models (Figure 5). Like the stacked-X model, the Cre-HJ structures are dyad-symmetric with two acute and two obtuse inter-arm angles. The assignment of continuous and crossing strands is less obvious for the Cre-bound junction, however, since both strands are discontinuous at the branch point. Based on the differences in tilt angles, phosphodiester torsion angles, and degree of unstacking, we refer to the strands bridging arms II-III and I-IV as 'continuous' and the strands bridging arms I-II and IIIIV as 'crossing', in keeping with current terminology. It is also clear that, like the stacked-X model, two crossover isomers are possible that correspond to swapping the roles between continuous and crossing strands. With respect to the overall Cre-DNA assembly, crossover isomerization could be readily accomplished by compressing the obtuse arm II-III and I-IV angles, opening the acute arm I-II and III-IV angles, and rotating the central phosphate linkages into opposite orientations (Figure 6). Because the Cre-Cre contacts in the HJ-bound tetramer are nearly fourfold-symmetric, only a small adjustment in the network of protein-protein and protein-DNA interactions is required to accomplish this rather subtle transition. The relationship between crossover isomer and direction of junction resolution is of central importance in understanding the function of HJ-resolving enzymes and this has 
been a central theme in recent studies of the Int family site-specific recombination pathway (Arciszewska et al., 1997; Azaro and Landy, 1997; Lee et al., 1998).

The Cre-HJ1 and Cre-HJ2 complexes share more gross architectural features with the square, low-salt form of the free HJ. Like the square junction, the arms of the Cre-HJ complexes lie approximately in the same plane and the bases at the branch point have become distinctly unstacked. In the case of the Cre-HJ2 complex, the DNA sequence of the junction that is formed is compatible with formation of a strictly fourfold-symmetric Cre-HJ complex. The fact that we observe a dyad-symmetric distortion from a fourfold-symmetric complex suggests that a fourfoldsymmetric structure may exist only as a transient intermediate during crossover isomerization and that the lower symmetry of both the covalent Cre-DNA intermediate (Guo et al., 1997) and the Cre-Holliday intermediates described here may have functional significance.

\section{Comparison with other protein-HJ complex studies}

The flexible nature of the $\mathrm{HJ}$ in solution is reflected in its ability to adopt different conformations when bound by junction-specific proteins (reviewed in White et al., 1997). RuvC, an enzyme that cleaves HJs in conjunction with RuvAB-mediated branch migration in bacteria (Dunderdale et al., 1991; Iwasaki et al., 1991, 1992; Tsaneva et al., 1992), binds to the junction as a dimer and introduces symmetry-related nicks in the pair of continuous strands (Bennett and West, 1995a). West and colleagues (Bennett and West, 1995b) have shown using electrophoresis of junctions with pairs of short and long arms that the RuvC-HJ complex is twofold-symmetric. Chemical probing of thymine bases with potassium permanganate also indicated that the crossover point forms an unstacked, open conformation. We find the pattern of electrophoretic mobilities and the evidence of solvent accessibility at the branch point in the RuvC-HJ complex to be remarkably consistent with the geometric and stereochemical features in the Cre-HJ1 and Cre-HJ2 complexes. Given the close relationship between the nearly planar dyad-symmetric Cre-bound HJ with the fourfoldsymmetric junction thought to exist in the RuvAB complex (Rice et al., 1997; Yu et al., 1997), it seems plausible that RuvC may bind to a HJ with a similar architecture as seen with Cre recombinase. Such a structure was in fact proposed in schematic form by Bennett and West (1995b).

In contrast to the RuvC-HJ complex, studies of the complexes of RuvA and phage T7 endonuclease I with synthetic HJs suggest that their structures differ from what we observe with Cre recombinase. RuvA binds HJs as a tetramer and recruits the hexameric ring helicase RuvB to drive branch migration (Tsaneva et al., 1992). In studies of RuvA-HJ complexes, Parsons et al. (1995) identified a fourfold-symmetric complex that was unstacked at the branch point, as expected for a square junction conformation. A still different complex was observed by Lilley and coworkers (Duckett et al., 1995) in a study of $\mathrm{T} 7$ endonuclease I, which binds and cleaves HJs as a dimer. In this case, the protein-junction complex is dyadsymmetric, but with apparent coaxial stacking of pairs of helical arms in a structure that appears more closely related to the stacked-X conformation of unbound junctions.

Within the $\lambda$-integrase family, XerC/XerD binding to synthetic HJs has been shown using permanganate probing to induce a pronounced unstacking of bases at the branch point relative to that observed in free junctions, indicating that these recombinases also change the unbound stackedX structure (Arciszewska et al., 1997). The same studies also showed that the branch point of the XerC/XerDbound junction is located at the center of the crossover region, as we observe in the Cre-HJ complexes. Recent studies in the Flp recombinase system have also shown that the recombinase unfolds HJ substrates and exposes bases at the branch point to solvent (Lee et al., 1998). Flp recombinase is the only Int family system for which electrophoretic mobilities and deduced symmetries of the recombinase-HJ complexes have been reported (Lee et al., 1998). Flp-HJ complexes show approximately fourfold symmetry, indicating a nearly planar arrangement of junction arms and either a complex with exact fourfold symmetry or one that readily converts between dyadsymmetric forms. Taken together with the recently established structural similarity of the proteins in the Int family (Guo et al., 1997; Hickman et al., 1997; Kwon et al., 1997; Subramanya et al., 1997), it seems likely that the XerC/XerD, $\lambda$-integrase, and Flp-HJ intermediate complexes are closely related to the Cre-HJ1 and Cre$\mathrm{HJ} 2$ structures described here.

\section{A model for strand cleavage-coupled $\mathrm{HJ}$ isomerization}

A central question in the Int family site-specific recombination pathway involves the asymmetry of cleavage of both the initial duplex substrates and of the Holliday intermediate. Only two of the four tyrosine nucleophiles are permitted to cleave the DNA substrates at each step. How is the appropriate pair of tyrosine residues activated and how is the alternative pair prevented from cleaving? In the resolution of the Holliday intermediate, the choice of which pair of tyrosine residues cleaves (or equivalently, which pair of DNA strands are cleaved) defines whether recombinant products or starting substrates will be formed. In early models of Int family site-specific recombination, branch migration of the Holliday intermediate between cleavage sites was suggested to be the switch responsible for deactivation of one pair of recombinases and activation of the other pair (Weisberg et al., 1983; Kitts and Nash, 1987; Nunes-Düby et al., 1987). This model was based on the requirement for homology between cleavage sites and it was assumed that branch migration would bring the second pair of scissile phosphates into mutual proximity to allow a second strand exchange and formation of recombinant products. In a more recent model of the Int family pathway, the optimal location of the Holliday intermediate branch point is limited to the central 1-2 bases of the crossover region, and homology between recombining sites is instead tested during the strand transfer step (Nunes-Düby et al., 1995). In this 'strandswapping' model, the switch between top and bottom strand cleavage required for resolution of the Holliday intermediate to form recombinants is linked to an isomerization of the $\mathrm{HJ}$ structure that results in the roles of the DNA strands. Both Int and the XerC/XerD 
recombinases have been shown to preferentially cleave the crossing strands in $\mathrm{HJ}$ substrates that have been biased to exist predominantly as a single crossover isomer in the unbound state. Using tethered junction arms (Arciszewska et al., 1997) and branch-point sequences designed to bias a single crossover isomer (Azaro and Landy, 1997), strand cleavage preference has been programmed by selecting the desired crossover isomer.

A related experiment was reported by Hoess et al. (1987) in a study of Cre mutants that accumulate Holliday intermediates but are unable to resolve them efficiently. Using a linear substrate containing two $\operatorname{lox} P$ sites, $\alpha$ structures were formed by the Cre mutants that could then be efficiently resolved to products by wild-type Cre. Resolution of the $\alpha$-structure gave primarily recombinant products, whereas resolution of a $\chi$-structure obtained by digestion of the loop connecting adjacent junction arms in the $\alpha$-structure gave an equal mixture of recombinants and starting substrates. In the context of the Int and XerC/ XerD studies, it seems likely that the Cre-catalyzed $\alpha$-structure effectively biased the Holliday intermediate towards a single crossover isomer. The $\chi$-structure in this case was presumably free to isomerize and populate both isomeric forms. Flp recombinase shares this apparent lack of resolution bias in studies with immobile Holliday substrates (Lee et al., 1998), yet the existence of two distinct kinetic forms of the Flp-HJ intermediate has been observed (Meyer et al., 1990).

The Cre-HJ1 and Cre-HJ2 complexes described here strongly support the idea that strand cleavage specificity is coupled to a HJ crossover isomer. We propose that crossover isomerization in the Cre-loxP pathway also involves an exchange in the roles of the DNA strands, but requires only subtle changes in quaternary structure and no restacking of the helical arms (Figure 6). An important feature of this mechanism is that the extensive interface $\left(\sim 5000 \AA^{2}\right.$ of buried protein surface) formed between the recombinase subunits in the synaptic tetramer would be preserved during isomerization. The energetic barrier to crossover isomerization may in fact be quite small for Cre recombinase. Assuming that the isomeric forms have roughly similar free energies, this would explain the lack of bias observed in the resolution of Holliday intermediates for this system and for the Flp system. In a kinetic analysis of Flp site-specific recombination, Meyer et al. (1990) were able to observe this isomerization process and showed that the interconversion of two Flp-HJ kinetic intermediates becomes rate-limiting at $0^{\circ} \mathrm{C}$.

The stereochemical coupling between strand cleavage and $\mathrm{HJ}$ isomer is likely to involve the peptide linker connecting the $\mathrm{C}$-terminal helix $\mathrm{N}$ to the catalytic tyrosinecontaining helix $\mathrm{M}$. Where helix $\mathrm{N}$ is exchanged between Cre protomers on arms that make an acute angle, the peptide linking helices $\mathrm{M}$ and $\mathrm{N}$ is well-ordered and adopts a compact conformation. In contrast, the peptide linking helices $\mathrm{M}$ and $\mathrm{N}$ across two arms that make an obtuse angle adopts a more extended, unrestrained conformation that may provide the required mobility for helix $M$ to initiate cleavage. This 'activated' active site is positioned on what we have termed the crossing strand in the Cre-HJ complex and corresponds to the same active site where a covalent $3^{\prime}$-phosphotyrosine linkage to a suicide DNA substrate was formed in a previous study (Guo et al., 1997). This arrangement is consistent with reports that Int and XerC/XerD both cleave the strand that is crossed in free Holliday substrates. We have assumed in these cases that the crossed strands in the protein-bound junctions maintain their identities as those bridging the arms that are closest in space, as in the stacked-X model and the Cre-HJ complex. The details of the stereochemical coupling between strand cleavage and $\mathrm{HJ}$ isomer are the subject of a separate study in this laboratory (F.Guo and G.Van Duyne, in preparation).

Although the isomerization model discussed above suggests a potential link between the stereochemical activation of a given pair of catalytic tyrosine residues and the isomeric state of the Cre-HJ complex, it does not address how cleavage preferences are related to the DNA sequences of the substrates. Int and Cre, for example, have been shown to proceed with a defined order of strand exchanges (Hoess et al., 1987; Kitts and Nash, 1988), which for Cre must be dictated initially by the DNA sequences in the recombining sites. Similarly, Landy and coworkers (Azaro and Landy, 1997) have demonstrated that specific HJ substrate isomers for Int can be dictated by the sequences present at the branch point. It is not clear from the Cre-HJ complexes why certain bases might prefer to adopt the crossing rather than continuous strand positions. Structural models of the recombinase proteins bound to their duplex substrates prior to cleavage and further studies of the Holliday intermediate complexes with wild-type sequences and at higher resolution should provide yet more pieces to the complex mechanistic puzzle in the Int family.

\section{Materials and methods}

\section{Preparation of HJs}

The HJ1 junction was formed from four 35-mer oligonucleotides using the sequences shown in Figure 1B. Oligonucleotides were synthesized by conventional phosphoramidite chemistry, and purified on a Hamilton PRP-1 reversed phase HPLC column with the dimethoxytrityl protecting group attached. Purified oligonucleotides were concentrated on a $2 \mathrm{ml}$ Q-sepharose column (Pharmacia), deprotected with $0.5 \%$ trifluoroacetic acid, and eluted with $20 \mathrm{mM}$ Tris- $\mathrm{HCl} \mathrm{pH} 7.8,1 \mathrm{M} \mathrm{NaCl}, 0.5 \mathrm{mM}$ EDTA. Following concentration and buffer exchange into $20 \mathrm{mM}$ Tris $\mathrm{pH} 7.8,50 \mathrm{mM} \mathrm{NaCl}, 0.5 \mathrm{mM}$ EDTA by Centricon-3 concentrators (Amicon), the concentrations of individual strands were determined by absorbance at $260 \mathrm{~nm}$ and used to prepare a stoichiometric mixture at $\sim 1 \mathrm{mM}$ concentration. The strands were annealed in a Perkin-Elmer 2400 thermal cycler using a linear $1 \%$ min gradient from $80-20^{\circ} \mathrm{C}$. The yield of four-way junction was $>95 \%$ as judged by the formation of a single band with low mobility on $22 \%$ non-denaturing polyacrylamide gels. The loxS6 sites were constructed by annealing the 15-mer 5'TATAACTTCGTATAG-3' and the 20-mer 5'-CATATGCTATACGAAGTTAT-3' following purification as described above.

\section{Protein purification and crystallization of Cre-HJ1 and Cre-HJ2}

The CreR173K mutant was generated by PCR mutagenesis using the wild-type expression construct in pET21a (Novagen) and the single-site mutation was verified by sequencing the entire coding region. Wildtype Cre and Cre R173K were purified, concentrated and stored as described (Guo et al., 1997). Crystals of both the HJ1 and HJ2 complexes were obtained by the hanging-drop vapor diffusion method. For Cre $\mathrm{R} 173 \mathrm{~K}-\mathrm{HJ} 1,4 \mu \mathrm{l}$ drops containing $50 \mu \mathrm{M}$ protein, $20 \mu \mathrm{M} \mathrm{HJ} 1,50 \mathrm{mM}$ sodium acetate $\mathrm{pH} 5.6,80 \mathrm{mM} \mathrm{MgCl}_{2}, 2 \mathrm{mM}$ dithiothreitol (DTT) and $22 \%$ 2-methyl-2,4-pentanediol (MPD) were equilibrated against $1 \mathrm{ml}$ reservoirs containing $50 \mathrm{mM}$ sodium acetate $\mathrm{pH}$ 5.0, 26\% MPD, $80 \mathrm{mM}$ $\mathrm{MgCl}_{2}$ at $18^{\circ} \mathrm{C}$. Thin plates $(150 \times 150 \times 20 \mu \mathrm{m})$ were obtained after two weeks. For Cre-HJ2, $6 \mu \mathrm{l}$ drops containing $65 \mu \mathrm{M}$ Cre, $81 \mu \mathrm{M}$ loxS6 
half-site, $25 \mathrm{mM}$ sodium acetate $\mathrm{pH} 5.7,12.5 \% \mathrm{MPD}, 5 \mathrm{mM} \mathrm{CaCl} \mathrm{C}_{2}$, $120 \mathrm{mM} \mathrm{NaCl}, 2.5 \mathrm{mM}$ DTT and $0.5 \mathrm{mM}$ spermine were equilibrated against $1 \mathrm{ml}$ reservoirs containing $50 \mathrm{mM}$ sodium acetate $\mathrm{pH} 5.0,24 \%$ MPD, $20 \mathrm{mM} \mathrm{CaCl}_{2}, 200 \mathrm{mM} \mathrm{NaCl}$. Thin plates grew to a maximum size of $200 \times 200 \times 30 \mu \mathrm{m}$ after one month at $4^{\circ} \mathrm{C}$.

\section{Data collection and structure determination}

Crystals were flash frozen in propane and stored in liquid nitrogen prior to data collection as described (Van Duyne et al., 1996). All diffraction data were measured at $100 \mathrm{~K}$ at the NSLS X25 beamline using a MAR research detector and processed using DENZO (Otwinowski, 1994). Cre-HJ1 crystals are orthorhombic, space group C222 ${ }_{1}, a=106.3, b=$ 122.7, $c=179.5 \AA$, with two Cre recombinase molecules and one half of the HJ in the asymmetric unit. Data are $99 \%$ complete to $2.7 \AA$, with $\mathrm{R}_{\mathrm{sym}}=0.072$ and average redundancy $=4.7$. Cre-HJ2 crystals are orthorhombic, space group $\mathrm{C} 222_{1}, a=106.9, b=122.5, c=180.0 \AA$. with two Cre molecules and one loxS6 site in the asymmetric unit. Data are $94 \%$ complete to $2.5 \AA$, with $\mathrm{R}_{\text {sym }}=0.053$ and average redundancy $=$ 2.8. Because Cre-HJ1 and Cre-HJ2 are nearly isomorphous with the Cre-loxA structure (Guo et al., 1997), both were determined by rigid body refinement of the Cre-lox $A$ domains at $3 \AA$, where each recombinase domain and the $13 \mathrm{bp}$ inverted repeat segment of the DNA sites were treated as rigid bodies. The C-terminal residues (316-341) of the recombinases and base pairs $1-4$ and $1^{\prime}-4^{\prime}$ were excluded from the rigid body refinement and were not included in the structure refinement until they could be unambiguously fit into unbiased $\sigma_{\mathrm{A}}$-weighted $2 \mathrm{~F}_{\mathrm{o}}-\mathrm{F}_{\mathrm{c}}$ electron density maps (Read, 1986).

\section{Refinement of Cre-HJ1 and Cre-HJ2}

The Cre-HJ1 and Cre-HJ2 structures were refined using torsion angle dynamics followed by conjugate gradients minimization in XPLOR (Rice and Brunger, 1996) at 3 and $2.7 \AA$, and for Cre-HJ2, at $2.5 \AA$ resolution. Refinement followed by model fitting to $\sigma_{\mathrm{A}}$-weighted $2 \mathrm{~F}_{\mathrm{O}}-$ $\mathrm{F}_{\mathrm{c}}$ maps using $\mathrm{O}$ (Jones et al., 1991) was repeated at each resolution.

A crystallographic dyad relates the two halves of the Cre-HJ1 complex, therefore the DNA in this structure is at least twofold disordered in the 4 bp adjacent to the branch point in each arm and in a single base pair located within the Cre binding site (Figure 1). Two models for disorder were considered. In model 1 , only one isomer of the $\mathrm{HJ}$ is present in the crystal: either the one shown in Figure 3 or the alternative isomer with arms II and III forming an acute angle. In either case, the Cre-HJ complex could pack in the crystal in two identical ways that are not influenced by the asymmetric base pairs and these bases would therefore be twofold disordered. In model 2, both isomers are equally populated; there are four equivalent ways to assign the arms of the junction, and the asymmetric bases are fourfold disordered. Because the minimal requirement of twofold disorder superimposes a purine and a pyrimidine at each asymmetric site, it was not possible to distinguish between these possibilities based on simple inspection of the electron density at $2.7 \AA$. The difficulty was compounded by the higher than average thermal parameters for DNA residues near the branch point. The models could also not be distinguished by comparing cross-validated $\mathrm{R}$-factors following refinement of the structures while imposing the appropriate non-crystallographic symmetry-constrained disorder model. The final refinement of the Cre-HJ1 complex was performed using a twofold disorder model where each of the asymmetric bases in the HJ1 arms are present at 50\% occupancy, using the arm and sequence assignments shown in Figures 1 and 3 . The final refinement model consists of residues 19-197 and 207-341 for Cre subunit A (bound to arm II), residues 19-197, 209-329 and 334-341 for subunit B (arm I), all DNA residues except the 5' thymidine on arms II and IV, 239 water molecules with good hydrogen bonding stereochemistry at unit occupancy and grouped thermal parameters (two per residue). The refinement for the Cre-HJ1 complex converged at $R=0.22$ and $R_{\text {free }}=0.29$ (5\% of data) using data to $2.7 \AA(\mathrm{F} \geqslant 2 \sigma)$ following a correction for the contribution of bulk solvent (Jiang and Brunger, 1994). R.m.s. deviations from ideal bond lengths and bond angles are $0.005 \AA$ and $0.95^{\circ}$, respectively.

The refinement of the Cre-HJ2 complex did not require a model for disorder, since the DNA sequence is twofold-symmetric. The final refinement model included residues 19-198, 210-275 and 283-341 for Cre subunit A, residues 19-198, 207-326 and 333-341 for subunit B, all DNA residues except the 5'-thymidine on arm II, 361 water molecules with good hydrogen bonding stereochemistry at unit occupancy, and tightly restrained individual thermal parameters. The refinement for the Cre-HJ2 complex converged at $R=0.20$ and $R_{\text {free }}=0.27(\mathrm{~F} \geqslant 2 \sigma)$ using data to $2.5 \AA$ following a bulk solvent correction. r.m.s. deviations from ideal bond lengths and bond angles are $0.007 \AA$ and $1.3^{\circ}$, respectively.

\section{Geometric calculations and illustrations}

DNA helicoidal parameters, torsion angles and curvature were computed using the program CURVES (Lavery and Sklenar, 1988). The angles between junction arms were defined as the angles between the principle moments of inertia of the duplex arm segments and were calculated using local programs. Figures 2, 3 and 5 were produced using RIBBONS (Carson, 1991); Figures 4 and 6 were produced with GRASP (Nicholls and Honig, 1993).

\section{Cre-HJ1 and Cre-HJ2 structures}

Coordinates for the Cre-HJ1 and Cre-HJ2 structures will be deposited with the Brookhaven Protein Data Bank under accession codes 3CRX and $2 \mathrm{CRX}$, respectively.

\section{Acknowledgements}

We thank Lonny Berman for access to and help with the NSLS X25 beamline, Jianping Ni for assistance with data collection and Mark Lemmon, Hillary Nelson, Art Landy and Simone Nünes-Duby for helpful suggestions with the manuscript. This work was supported by NIH grant GM-55041 and an American Cancer Society Institutional Research Grant to the University of Pennsylvania.

\section{References}

Abremski,K.E. and Hoess,R.H. (1992) Evidence for a second conserved arginine residue in the integrase family of recombination proteins. Protein Eng., 5, 87-91.

Abremski,K., Hoess,R. and Sternberg,N. (1983) Studies on the properties of P1 site-specific recombination: evidence for topologically unlinked products following recombination. Cell, 32, 1301-1311.

Arciszewska,L., Grainge,I. and Sherratt,D. (1995) Effects of Holliday junction position on Xer-mediated recombination in vitro. EMBO J., 14, 2651-2660.

Arciszewska,L.K., Grainge,I. and Sherratt,D.J. (1997) Action of sitespecific recombinases XerC and XerD on tethered Holliday junctions. EMBO J., 16, 3731-3743.

Argos,P. et al. (1986) The integrase family of site-specific recombinases: regional similarities and global diversity. EMBO J., 5, 433-440.

Azaro,M.A. and Landy,A. (1997) The isomeric preference of Holliday junctions influences resolution bias by lambda integrase. EMBO J., 16, 3744-3755.

Bennett,R.J. and West,S.C. (1995a) RuvC protein resolves Holliday junctions via cleavage of the continuous (noncrossover) strands. Proc. Natl Acad. Sci. USA, 92, 5635-5639.

Bennett,R.J. and West,S.C. (1995b) Structural analysis of the RuvCHolliday junction complex reveals an unfolded junction. J. Mol. Biol., 252, 213-226.

Blakely,G., May,G., McCulloch,R., Arciszewska,L.K., Burke,M., Lovett,S.T. and Sherratt,D.J. (1993) Two related recombinases are required for site-specific recombination at dif and cer in E.coli K12. Cell, 75, 351-361.

Burgin,A.B. and Nash,H.A. (1995) Suicide substrates reveal properties of the homology-dependent steps during integrative recombination of bacteriophage lambda. Curr. Biol., 5, 1312-1321.

Campbell,A. (1962) Episomes. Adv. Genet., 11, 101-145.

Carson,M. (1991) Ribbons 2.0. J. Appl. Crystallogr., 24, 958-961.

Cheng,C., Kussie,P., Pavletich,N. and Shuman,S. (1998) Conservation of structure and mechanism between eukaryotic topoisomerase I and site-specific recombinases. Cell, 92, 841-850.

Churchill,M.E.A., Tullius,T.D., Kallenbach,N.R. and Seeman,N.C. (1988) A Holliday recombination intermediate is twofold symmetric. Proc. Natl Acad. Sci. USA, 85, 4653-4656.

Clegg,R.M., Murchie,A.I.H. and Lilley,D.M.J. (1994) The solution structure of the four-way DNA junction at low-salt conditions: a fluorescence resonance energy transfer analysis. Biophys. J., 66, 99-109.

Craig,N.L. (1988) The mechanism of conservative site-specific recombination. Annu. Rev. Genet., 22, 77-105.

de Massey,B., Studier,F.W., Dorgai,L., Appelbaum,F. and Weisberg,R.A. (1984) Enzymes and the sites of genetic recombination: studies with gene- 3 endonuclease of phage T7 and with site-affinity mutants of phage lambda. Cold Spring Harbor Symp. Quant. Biol., 49, 715-726. 
Dixon,J.E. and Sadowski,P.D. (1993) Resolution of synthetic chi structures by the FLP site-specific recombinase. J. Mol. Biol., 234, 522-533.

Duckett,D.R., Murchie,A.I., Diekmann,S., von,K.E., Kemper,B. and Lilley,D.M. (1988) The structure of the Holliday junction, and its resolution. Cell, 55, 79-89.

Duckett,D.R., Panis,M.E.J. and Lilley,D.M.J. (1995) Binding of the junction-resolving enzyme bacteriophage T7 endonuclease I to DNA: separation of binding and catalysis by mutation. J. Mol. Biol., 246 , 95-107.

Dunderdale,H.J., Benson,F.E., Parsons,C.A., Sharples,G.J., Lloyd,R.G. and West,S.C. (1991) Formation and resolution of recombination intermediates by E.coli RecA and RuvC proteins. Nature, 354, 506-510.

Elborough,K.M. and West,S.C. (1990) Resolution of synthetic Holliday junctions in DNA by an endonuclease from calf thymus. EMBO J., 9, 2931-2936.

Futcher,A.B. (1986) Copy number amplification of the 2 micron circle plasmid of Saccharomyces cerevisiae. J. Theor. Biol., 119, 197-204.

Goodsell,D.S., Grzeskowiak,K. and Dickerson,R.E. (1995) Crystal structure of C-T-C-T-C-G-A-G-A-G. Implications for the structure of the Holliday junction. Biochemistry, 34, 1022-1029.

Guo,F., Gopaul,D.N. and Van Duyne,G.D. (1997) Structure of Cre recombinase complexed with DNA in a site-specific recombination synapse. Nature, 389, 40-46.

Hickman,A.B., Waninger,S., Scocca,J.J. and Dyda,F. (1997) Molecular organization in site-specific recombination: the catalytic domain of bacteriophage HP1 integrase at $2.7 \AA$ resolution. Cell, 89, 227-237.

Hoess,R.H., Ziese,M. and Sternberg,N. (1982) P1 site-specific recombination: nucleotide sequence of the recombining sites. Proc. Natl Acad. Sci. USA, 79, 3398-3402.

Hoess,R., Wierzbicki,A. and Abremski,K. (1987) Isolation and characterization of intermediates in site-specific recombination. Proc. Natl Acad. Sci. USA, 84, 6840-6844.

Holliday,R. (1964) A mechanism for gene conversion in fungi. Genet. Res., 5, 282-304.

Iwasaki,H., Takahagi,M., Shiba,T., Nakata,A. and Shinagawa,H. (1991) Escherichia coli RuvC protein is an endonuclease that resolves the Holliday structure. EMBO J., 10, 4381-4389.

Iwasaki,H., Takahagi,M., Nakata,A. and Shinagawa,H. (1992) Escherichia coli RuvA and RuvB proteins specifically interact with Holliday junctions and promote branch migration. Genes Dev., 6, 2214-2220.

Jiang,J.S. and Brunger,A.T. (1994) Protein hydration observed by X-ray diffraction: solvation properties of penicillopepsion and neuraminidase crystal structures. J. Mol. Biol., 243, 100-115.

Jones,T.A., Zou,J.Y., Cowan,S.W. and Kjeldgaard,M. (1991) Improved methods for building protein models in electron density maps and the location of errors in these models. Acta Cryst., A47, 110-119.

Kallenbach,N.R., Ma,R. and Seeman,N.C. (1983) An immobile nucleic acid junction constructed from oligonucleotides. Nature, 305, 829-831.

Kitts,P.A. and Nash,H.A. (1987) Homology-dependent interactions in phage lambda site-specific recombination. Nature, 329, 346-348.

Kitts,P.A. and Nash,H.A. (1988) Bacteriophage lambda site-specific recombination proceeds with a defined order of strand exchanges. $J$. Mol. Biol., 204, 95-107.

Kleckner,N. (1996) Meiosis: how could it work? Proc. Natl Acad. Sci. USA, 93, 8167-8174.

Kleff,S., Kemper,B. and Sternglanz,R. (1992) Identification and characterization of yeast mutants and the gene for a cruciform cutting endonuclease. EMBO J., 11, 699-704.

Kowalczykowski,S.C., Dixon,D.A., Eggleston,A.K., Lauder,S.D. and Rehrauer,W.M. (1994) Biochemistry of homologous recombination in Escherichia coli. Microbiol. Rev., 58, 401-465.

Kwon,H.J., Tirumalai,R., Landy,A. and Ellenberger,T. (1997) Flexibility in DNA recombination: structure of the lambda integrase catalytic core. Science, 276, 126-131.

Landy,A. (1989) Dynamic, structural, and regulatory aspects of lambda site-specific recombination. Annu. Rev. Biochem., 58, 913-949.

Lavery,R. and Sklenar,H. (1988) The definition of generalised helicoidal parameters and of axis curvature for irregular nucleic acids. J. Biomol. Struct. Dynam., 6, 63-91.

Lee,J. and Jayaram,M. (1995) Role of partner homology in DNA recombination. Complementary base pairing orients the $5^{\prime}$-hydroxyl for strand joining during Flp site-specific recombination. J. Biol. Chem., 270, 4042-4052.
Lee,J., Lee,J. and Jayaram,M. (1995) Junction mobility and resolution of Holliday structures by Flp site-specific recombinase. Testing partner compatibility during recombination. J. Biol. Chem., 270, 19086-19092.

Lee,J., Voziyanov,Y., Pathania,S. and Jayaram,M. (1998) Structural alterations and conformational dynamics in Holliday junctions induced by binding of a site-specific recombinase. Mol. Cell., 1, 483-494.

Lilley,D.M.J. and Clegg,R.M. (1993) The structure of the four-way junction in DNA. Annu. Rev. Biophys. Biomol. Struct., 22, 299-328.

Lu,M., Guo,Q., Seeman,N.C. and Kallenbach,N.R. (1991) Parallel and antiparallel Holliday junctions differ in structure and stability. J. Mol. Biol., 221, 1419-1432.

Meselson,M. (1972) Formation of hybrid DNA by rotary diffusion during genetic recombination. J. Mol. Biol., 71, 795-798.

Meyer,L.L., Inman,R.B. and Cox,M.M. (1990) Characterization of Holliday structures in FLP protein-promoted site-specific recombination. Mol. Cell. Biol., 10, 235-242.

Miick,S.M., Fee,R.S., Millar,D.P. and Chazin,W.J. (1997) Crossover isomer bias is the primary sequence-dependent property of immobilized Holliday junctions. Proc. Natl Acad. Sci. USA, 94, 9080-9084.

Mizuuchi,K., Kemper,B., Hays,J. and Weisberg,R.A. (1982) T4 endonuclease VII cleaves Holliday structures. Cell, 29, 357-365.

Nicholls,A. and Honig,B. (1993) GRASP. Columbia University, New York.

Nunes-Düby,S., Matsumoto,L. and Landy,A. (1987) Site-specific recombination intermediates trapped with suicide substrates. Cell, $\mathbf{5 0}$ 779-788.

Nunes-Düby,S., Azaro,M.A. and Landy,A. (1995) Swapping DNA strands and sensing homology without branch migration in lambda sitespecific recombination. Curr. Biol., 5, 139-148.

Nunes-Düby,S.E., Yu,D. and Landy,A. (1997) Sensing homology at the strand-swapping step in lambda excisive recombination. J. Mol. Biol., 272, 493-508.

Nunes-Düby,S.E., Tirumalai,R.S., Kwon,H.J., Ellenberger,T. and Landy,A. (1998) Similarities and differences among 105 members of the Int family of site-specific recombinases. Nucleic Acids Res., 26, 391-406.

Otwinowski,Z. (1994) DENZO and SCALEPACK. Yale University, New Haven, CT.

Parsons,C.A., Kemper,B. and West,S.C. (1990) Interaction of a fourway junction in DNA with T4 endonuclease VII. J. Biol. Chem., $\mathbf{2 6 5}$ 9285-9289.

Parsons,C.A., Stasiak,A., Bennett,R.J. and West,S.C. (1995) Structure of a multisubunit complex that promotes DNA branch migration. Nature, 374, 375-378.

Read,R.J. (1986) Improved Fourier coefficients for maps using phases from partial structures with errors. Acta Cryst., A42, 140-149.

Rice,L.M. and Brunger,A.T. (1996) Torsion angle dynamics: reduced variable confomational sampling enhances crystallographic structure refinement. Proteins Struct. Func. Genet., 19, 277-290.

Rice,D.W., Rafferty,J.B., Artymiuk,P.J. and Lloyd,R.G. (1997) Insights into the mechanisms of homologous recombination from the structure of RuvA. Curr. Opin. Struct. Biol., 7, 798-803.

Sadowski,P.D. (1995) The Flp recombinase of the 2-microns plasmid of Saccharomyces cerevisiae. Prog. Nucleic Acid Res. Mol. Biol., 51, 53-91.

Seeman,N.C. and Kallenbach,N.R. (1994) DNA branched junctions. Annu. Rev. Biophys. Biomol. Struct., 23, 53-86.

Sekiguchi,J., Seeman,N.C. and Shuman,S. (1996) Resolution of Holliday junctions by eukaryotic DNA topoisomerase I. Proc. Natl Acad. Sci. USA, 93, 785-789.

Sharples,G.J., Chan,S.N., Mahdi,A.A., Whitby,M.C. and Lloyd,R.G. (1994) Processing of intermediates in recombination and DNA repair: identification of a new endonuclease that specifically cleaves Holliday junctions. EMBO J., 13, 6133-6142.

Sherratt,D.J., Arciszewska,L.K., Blakely,G., Colloms,S., Grant,K., Leslie,N. and McCulloch,R. (1995) Site-specific recombination and circular chromosome segregation. Phil. Trans. R. Soc. Lond. Ser. B, 347, 37-42.

Sigal,N. and Alberts,B. (1972) Genetic recombination: the nature of crossed strand-exchange between two homologous DNA molecules. J. Mol. Biol., 71, 789-793.

Stark,W.M., Boocock,M.R. and Sherratt,D.J. (1989) Site-specific recombination by Tn3 resolvase. Trends Genet., 5, 304-309.

Sternberg,N., Hamilton,D., Austin,S., Yarmolinsky,M. and Hoess,R. (1981) Site-specific recombination and its role in the life cycle of bacteriophage P1. Cold Spring Harbor Symp. Quant. Biol., 1, 297-309. 
Stewart,L., Redinbo,M.R., Qiu,X., Hol,W.G.J. and Champoux,J.J. (1998) A model for the mechanism of human topoisomerase I. Science, 279, 1534-1540.

Subramanya,H.S., Arciszewska,L.K., Baker,R.A., Bird,L.E., Sherratt,D.J. and Wigley,D.B. (1997) Crystal structure of the site-specific recombinase, XerD. EMBO J., 16, 5178-5187.

Symington,L. and Kolodner,R. (1985) Partial purification of an endonuclease from Saccharomyces cerevisiae that cleaves Holliday junctions. Proc. Natl Acad. Sci. USA, 82, 7247-7251.

Tsaneva,I.R., Muller,B. and West,S.C. (1992) ATP-dependent branch migration of Holliday junctions promoted by RuvA and RuvB proteins of E.coli. Cell, 69, 1171-1180.

Van Duyne,G.D., Ghosh,S., Maas,W.K. and Sigler,P.B. (1996) Structure of the oligomerization and L-arginine binding domain of the arginine repressor of Escherichia coli. J. Mol. Biol., 256, 377-391.

von Kitzing,E., Lilley,D.M.J. and Diekmann,S. (1990) The stereochemistry of a four-way DNA junction: a theoretical study. Nucleic Acids Res., 18, 2671-2683.

Weisberg,R. and Landy,A. (1983) Site-specific recombination in phage lambda. In Hendrix, R.W., Roberts,J.W., Stahl,F.W. and Weisberg,R.A. (eds), Lambda II. Cold Spring Harbor Laboratory Press, Cold Spring Harbor, NY, pp. 211-250.

Weisberg,R.A., Enquist,L.W., Foeller,C. and Landy,A. (1983) A role for DNA homology in site-specific recombination: the isolation and characterization of a site affinity mutant of coliphage lambda. $J$. Mol. Biol., 170, 319-342.

West,S.C. and Korner,A. (1985) Cleavage of cruciform DNA structures by an activity from Saccharomyces cerevisiae. Proc. Natl Acad. Sci. USA, 82, 6445-6449.

White,M.F., Giraud-Panis,M.J., Pohler,J.R. and Lilley,D.M. (1997) Recognition and manipulation of branched DNA structure by junctionresolving enzymes. J. Mol. Biol., 269, 647-664.

Wierzbicki,A., Kendall,M., Abremski,K. and Hoess,R. (1987) A mutational analysis of the bacteriophage $\mathrm{P} 1$ recombinase Cre. J. Mol. Biol., 195, 785-794.

Wood,A.A., Nunn,C.M., Trent,J.O. and Neidle,S. (1997) Sequencedependent crossed helix packing in the crystal structure of a B-DNA dodecamer yields a detailed model for the Holliday junction. J. Mol. Biol., 269, 827-841.

Yu,X., West,S.C. and Egelman,E.H. (1997) Structure and subunit composition of the RuvAB-Holliday junction complex. J. Mol. Biol., 266, 217-222.

Zhu,X.D., Pan,G., Luetke,K. and Sadowski,P.D. (1995) Homology requirements for ligation and strand exchange by the FLP recombinase. J. Biol. Chem., 270, 11646-11653.

Received May 18, 1998; accepted May 22, 1998 